IEEE Instrumentation and Measurement

Technology Conference

Baltimore, MD, USA, May 1-4, 2000

\title{
Odor-Based Incontinence Sensor
}

\author{
Huadong $\mathrm{Wu}^{1}$ and Mel Siegel ${ }^{2}$ \\ Robotics Institute, School of Computer Science \\ Carnegie Mellon University, Pittsburgh, PA 15213 \\ Phone: 412-268-8802; fax: 412-268-5569 \\ E-mail: ${ }^{1}$ whd@ @cs.cmu.edu; ${ }^{2}$ mws@cs.cmu.edu, \\ WWW: ${ }^{1}$ http://www.cs.cmu.edu/ whd; ${ }^{2}$ http://www.cs.cmu.edu/ mws
}

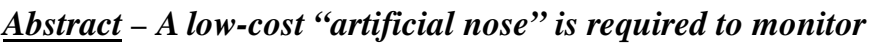
incontinence of elderly patients in nursing homes. With the aim of identifying a small array of inexpensive sensors whose response vector could provide an unambiguous signature at a useful sensitivity level, we characterized the sensitivity of seven easily available solid-state sensors to fecal component gases and vapors, and to potential interferences anticipated in the environment. The sensors' dynamic responses in a rapid periodic heating and cooling cycle proved substantially quieter than their $D C$ responses at constant temperatures. However, large sensor-to-sensor variability combined with undesirably high sensitivity to humidity proved so vexing that the practical prospects for this approach were deemed discouraging. An alternative approach using the differential response of a matched pairs of sensors, with one of the pair equipped with a filter that traps fecal component gases and vapors, is now under investigation.
\end{abstract}

\section{INTRODUCTION}

\section{A. Background}

Rapid advances in the development of life-prolonging medicines and medical procedures has led to an aging population, and to a corresponding increase in the number of nursing home patients, many of whom are incontinent. When incontinence is not attended to quickly, patients experience rapid degeneration of skin health, making them prone to pressure ulcers (formerly called "bedsores") in regions that remain in contact with fecal matter [1]. Inasmuch as many of these patients are demented as well as incapacitated, they cannot summon assistance, so the attendant staff must be relied on to detect and remedy the problem promptly; at the same time, the economics of our current medical system leads to staff deficiencies in number and in diligence. Although it is easy for a conscientious attendant to detect the characteristic odor of a diaper that needs to be changed, in practice it often happens that nobody is around to notice for several hours - especially late at night - by which time difficult-to-reverse skin damage has begun.

\section{B. Components of Fecal Odor}

Feces are composed largely of ingested materials that are not digested, e.g., cellulose fiber and other roughage, together with water, salts, mucus, cellular debris sloughed off from the intestines, and bacteria [1]. The pungent odor of feces is due to a complex mixture of compounds produced by bacterial action, primarily on amino acids (protein building blocks). The odoriferous products include indole, skatole, mercaptans (methyl sulfides: methane thiol, dimethyl disulfide, and dimethyl trisulfide, etc.), hydrogen sulfide, and ammonia. Also present are gases that are odorless to humans, e.g., methane and hydrogen; these are nevertheless potential targets for an "artificial nose".

The benzopyrrole volatiles indole $\left(\mathrm{C}_{8} \mathrm{H}_{7} \mathrm{~N}\right)$ and skatole $\left(\mathrm{CH}_{3}-\right.$ $\mathrm{C}_{7} \mathrm{H}_{6} \mathrm{~N}$ ), principally from the digestion of the amino acid tryptophen [1], were until a decade ago believed to be the most characteristic components of human feces odor. Moore et al. [4] then demonstrated with human observers and GCMS analysis that although the components responsible for fecal odor are complex and are to some extent influenced by dietary and endogenous contributions, the principal components of the fecal signature detected by the human nose are the methyl sulfides ("mercaptans") rather than the less volatile benzopyrroles like skatole and indole. This is not to say that response to benzopyroles might not become the primary basis for an artificial sensor.

Mercaptans are simple organic molecules with alcohol-like structures, wherein the alcohol's - $\mathrm{OH}$ is replaced by a thiol's -SH, e.g., $\mathrm{CH}_{3}-\mathrm{SH}$ (methane thiol, or methyl mercaptan), $\mathrm{CH}_{3}-\mathrm{S}-\mathrm{S}-\mathrm{H}_{3} \mathrm{C}$ (dimethyl sulfide, or dimethyl disulfide), and $\mathrm{CH}_{3}-\mathrm{S}-\mathrm{S}-\mathrm{S}-\mathrm{H}_{3} \mathrm{C}$ (dimethyl trisulfide). The mercaptans have very strong disagreeable odors and are very permeable; they are widely used to add a leak-warning odor to otherwise odorless gases, e.g., "natural gas", which is primarily methane. Solid-state gas sensors nominally optimized for detecting natural gas tagged with mercaptans have been marketed in the past, but they are not currently available. 


\section{Odor-Sensor Background}

There are no commercially available sensors specifically targeting odors that are "unpleasant"1 to the human olfactory sense. The closest consumer devices are the solid-state sensor based alarms sold for warning of leaks of domestic fuel gases (required by law in Japan), such as methane, and propane, the incompletely burned hydrocarbon fuel product carbon monoxide, and miscellaneous volatile hydrocarbons (VHCs) that may make their way into the environment via evaporation of solvents, etc. Since the sensors in these consumer devices generally respond to reducing gases (i.e., fuels vs. oxidizers), and alcohols are fuels, and mercaptans are analogs of alcohols, we generally expect the same classes of sensors to be sensitive to mercaptans too. This is supported by the fact that similar or identical solid state sensors are used in simple breath alcohol detectors used by police, in coin-operated machines in taverns, and available to consumers in hand-held instruments.

The core element of the most common type of solid-state sensor, the "Taguchi Gas Sensor" (TGS), is a sintered metal oxide. It detects gases because its bulk electrical conductivity changes when a reducing gas is absorbed on the metal oxide surfaces. The basic principle is illustrated specifically for the very common tin oxide $\left(\mathrm{SnO}_{2}\right)$ TGS. The oxide is normally produced in a slightly reduced state, e.g., $\mathrm{SnO}_{2-\varepsilon}$, making it a semiconductor. If the sensor is heated to a high temperature, e.g. $400{ }^{\circ} \mathrm{C}$, in the relative absence of oxygen, free electrons can flow easily through the grain boundaries. But in clean air, oxygen is absorbed onto the tin dioxide particle surface, forming a potential barrier at the grain boundaries; this can also be visualized as the capture of electrons via the relatively high electron affinity of oxygen, or as a reduction in the value of $\varepsilon$ in the formula $\mathrm{SnO}_{2-\varepsilon}$, making the oxide "less metallic". However it is visualized, the result is an increase in electrical resistance with increasing partial pressure of oxygen, and a decrease in electrical resistance with increasing partial pressure of reducing gas.

The precise details depend also on operating conditions, e.g., sensor temperature, the oxide's physical processing, e.g., grain size and packing, and the material's chemical modifications, e.g., via the addition of small quantities of precious metals which catalyze specific reactions. Basically, it is through these details that one sensor is sold in numerous models, each nominally optimized for either a specific gas target, or as a general purpose sensor for a family of chemically related gases.

The prospect is thus raised that the outputs of an array of broadly general-purpose sensors, each with a small selectivity

\footnotetext{
1 "Unpleasant" is not an entirely appropriate term in this context, inasmuch as many chemicals, e.g., musk, are regarded as pleasant at extremely low concentrations but unpleasant at higher concentrations.
}

bias induced by a production or an operating parameter, can be combined by a signature recognition algorithm to synthesize a relatively high degree of selectivity [5]. In practice, the construction of an appropriate algorithm is hampered by the extreme non-linearity of the response functions, and by the existence of strong cross-sensitivities (the sensitivity to gas A depends on the presence of gas B), which makes calibration (e.g., training a neural network) exceedingly difficult.

The relative straightforwardness of the fecal odor detection problem nevertheless stimulates us to see if we can develop a practical and economical "artificial nose" whose sensitivity and selectivity are attuned to the problem of incontinence in nursing homes. This sensor's design, packaging, and "human interface" would have to fit comfortably into the nursing home culture and environment, at an initial and ongoing cost that makes it commensurate with other nursing home apparatus of similar medical value.

\section{EXPERIMENTAL DESIGN}

\section{A. Samples and Air-flow Control}

We tested the response of several sensor types to indole, skatole, and dimethyl disulfide vapors, to hydrogen sulfide gas, and to other feces indicating vapors and gases, and to several potentially interfering vapors and gases, separately and in binary and tertiary combinations. The potentially interfering vapors and gases were chemical agents commonly used in nursing home environments, e.g., ammonia, Clorox bleach, isopropanol, and iodine. The ammonia is of interest both as a cleaning product and as a breakdown product of urine.

The major components of our apparatus are airflow control, sample vapor generation and dilution, computer-based data acquisition and control. The key requirements are to produce air with known odiferous components at known concentrations, and to deliver it to the sensors on demand. The airflow and sample delivery system is illustrated in Figure 1.

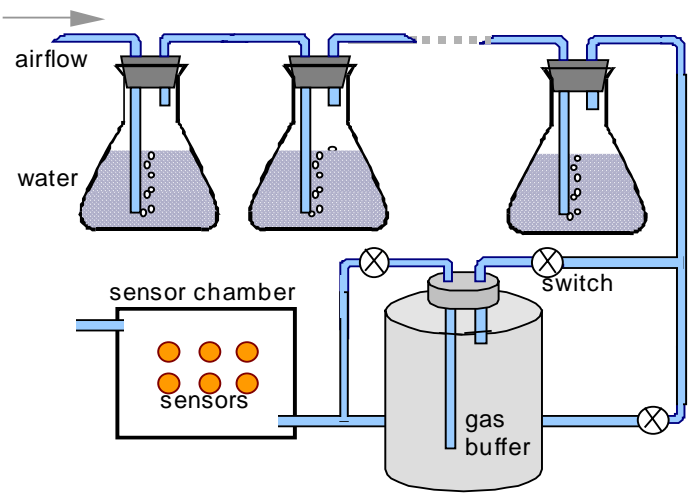

Figure 1. Airflow system for gas sensor testing 
The airflow comes from a central compressed air supply ("house air"). We add several stages of oil mist and drying filters, and the required airflow regulators and flow rate meters. We can control and measure the airflow from $\sim 0.5$ liter per minute up to 20 liter per minute. The clean and dry air is bubbled sequentially through water in several flasks, allowing us to control the humidity (degree of saturation) by controlling the duration of contact between air and water and by controlling of water temperature. Because we observed very strong sensitivity and cross sensitivity of the sensors to water vapor, we were cautious to stabilize the humidity at the sensors per se. This was done via a pair of humidity sensors installed in the sensor chamber. [Regrettably, it was not possible within the scope of the present experiments for the humidity sensors to be calibrated precisely, nor could their sensitivities to other chemicals under test be measured precisely.]

The test chamber is a gasketed stainless steel box of $200 \mathrm{~mm}$ x $150 \mathrm{~mm}$ x $105 \mathrm{~mm}$, volume 3.15 liter. The time-constant for exponential buildup or decay of sample concentration in the chamber is this volume divided by the flow rate. For example, when the airflow is 4.72 liter per minute, it requires 40 seconds for the sample concentration in the chamber to reach $\mathrm{e}^{-1}$ of its concentration in the incoming air stream, and conversely, 40 seconds to fall to $\mathrm{e}^{-1}$ of its initial concentration in the chamber when the sample flow is replaced by a pure air flow. In each experiment, we assure that the humidity of the sample-bearing stream and the "pure air" stream are the same, and the humidity of the chamber is first stabilized to this value.

The sample streams of some of the low vapor pressure solid samples are generated by slow passage of air through a buffer volume, an 18.9 liter bottle, equilibrated to the material's saturation vapor pressure at room temperature. Since the bottle contains an excess of the solid, as long as the flow rate through the bottle is small the exiting stream is saturated. This stream is further diluted by mixing with a (typically much) larger flow of pure air. The two airstreams are equally humidified.

The sample streams of the higher vapor pressure liquid samples are generated by slow exponential dilution by passing air through an 18.9 liter bottle that has been prepared by injection, with an airtight syringe, of a measured volume of the headspace over the liquid. The sample and air are allowed adequate time to equilibrate before the exponential dilution is begun. In this case, also, care is taken to assure that the humidity is the same in the sample and diluting streams, etc.

Vapor pressure data for most of the materials of interest to us are extremely sparse. For example, for methyl disulfide we have, from the Beilstein Reference [8], only two points: 6.7 torr at $0.0 \mathrm{C}$ and 1268 torr at $128.6 \mathrm{C}$ [1 torr $=1333$ pascal]. Fortunately, from these end points we can estimate the empirical constants $a$ and $b$ in the equation [6]

$$
\log _{10} p=-(0.05223 / T) a+b,
$$

from which the headspace vapor pressure can be estimated at intermediate temperatures. For methyl disulfide at room temperature, the estimated headspace vapor pressure is 20.5 torr. Atmospheric pressure at the altitude of our laboratory is 742 torr. Thus when we withdraw $1 \mathrm{ml}$ of headspace vapor and inject it into an 18.9 liter bottle we estimate the molar concentration of the equilibrated mixture to be (20.5 torr/742 torr $)^{*}\left(10^{-3}\right.$ liter $/ 18.9$ liter $)=1.46 \mathrm{ppm}$. Analogous estimates are made for the other sample types.

\section{B. Sensor Control and Measurement}

Figaro TGS solid-state sensors are widely used for reducing gas detection, organic solvent detection, etc. They are inexpensive, and in principle require only a power supply for heating and a resistance-measuring instrument for sensing. We examined six Figaro models: TGS2600, TGS2610, TGS1611, TGS2620, TGS825 and TGS826. Figaro's specification of their preferred applications is shown in Table 1.

Table 1. Target gases for Figaro sensors

\begin{tabular}{|ll|}
\hline Figaro sensor type & most sensitive to: \\
TGS2600 & general air contaminants \\
TGS2610 & general hydrocarbons \\
TGS2611 & methane natural gas \\
TGS2620 & alcohol and organic solvent vapors \\
TGS825 & hydrogen sulfide \\
TGS826 & ammonia \\
\hline
\end{tabular}

\section{(a). Static Response}

We measure (via the analog-to-digital converter inputs of a Keithley KPCI3108 computer data acquisition and control board) the sensor resistance using the simple voltage divider shown in Figure 2.

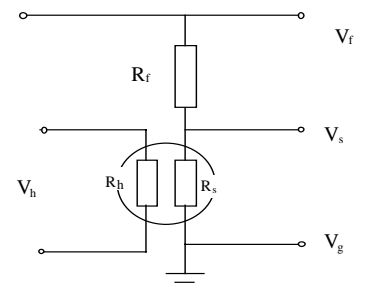

Figure 2. Figaro sensor gas detection circuit

$R_{h}$ is the resistive heater, across which voltage $V_{h}$ is imposed. $R_{S}$ is the tin oxide film whose resistance varies according to heater temperature and ambient gas composition. We measure $R_{s}=R_{f} V_{s} /\left(V_{f}-V_{s}\right)$, where as $\mathrm{R}_{\mathrm{f}}$ is chosen to be approximately the same as $R_{\mathrm{S}}$ at a low concentration of a gas of interested. Since $\Delta V_{S} / V_{S}=\Delta R_{S} /\left(R_{S}+R_{f}\right)$ this choice is a reasonable compromise between sensitivity and dynamic range.

\section{(b). Dynamic Response}


In typical Figaro sensor applications, the measurement is static, i.e., data are taken only when the sensors' tin oxide film is in its steady hot state. Since the long-term stability of Taguchi-type sensors is notoriously poor, we instead investigated using the dynamic response, i.e., temporal analysis of the signal when the sensor is thermally cycled at a rate up to about 5 times longer of its thermal time constant. Several potential advantages can be imagined:

- overcoming drift and low frequency noise

- self cleaning (if the temperature periodically becomes high enough to evaporate adsorbed sample)

- using the time constant for resistance change as a signature of gas composition, etc.

Figaro sensors use a filament heater and a relatively massive ceramic substrate to carry the tin oxide film. They thus have a relatively long thermal time constant, the order of 3 seconds. On the other hand, the MicroChemical Systems sensors [7], which are made using microelectronic integrated circuit fabrication technology, integrate the tin oxide thin film with a heater embedded in the silicon substrate. This gives them a thermal time constant the order of 20 milliseconds, about 150 times faster than the Figaro sensors.

To examine the dynamic response of both Figaro and MicroChemical Systems sensors we use the heating circuit illustrated in Figure 3 to excite two sensors of each type in parallel: MicroChemical sensors on the left and Figaro sensors on the right.

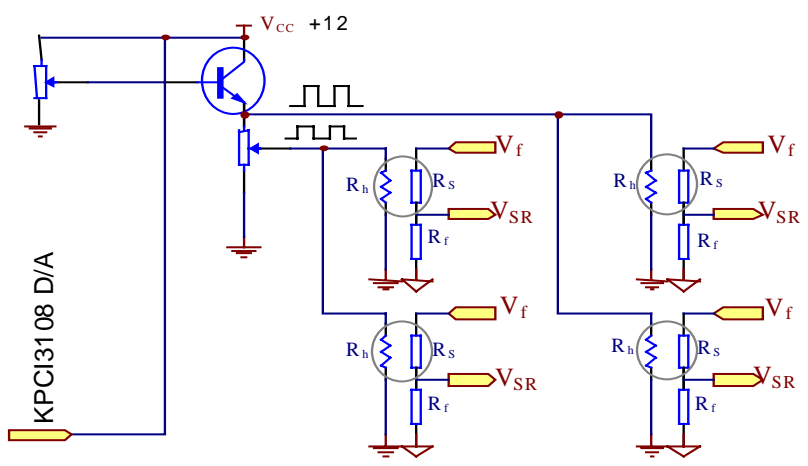

Figure 3. Dynamic response measurement circuit for Figaro (right) and MicroChemical (left) sensors

The heater voltage is controlled by the KPCI3108 D/A output buffered by a power transistor. The TGS2600s receive a high heating voltage of 5.00 volts and low cooling down voltage of 1.10 volts. The MicroChemical Systems sensors receive a high heating voltage of 2.35 volts and low cooling down voltage of 0.50 volts.

\section{(c). Dynamic Data Collection Protocol}

Although we anticipate that in the future we may take advantage of the fast cycling capability of the MicroChemical Systems sensors, in the initial experiments we accommodate them to the longer time constant of the Figaro sensors. Figure
4 illustrates 15 second hot, and 15 second cool cycle that we use. We identify four phases: (1) cool to hot transition phase, (2) steady hot phase, (3) hot to cool phase, and (4) steady cool phase.

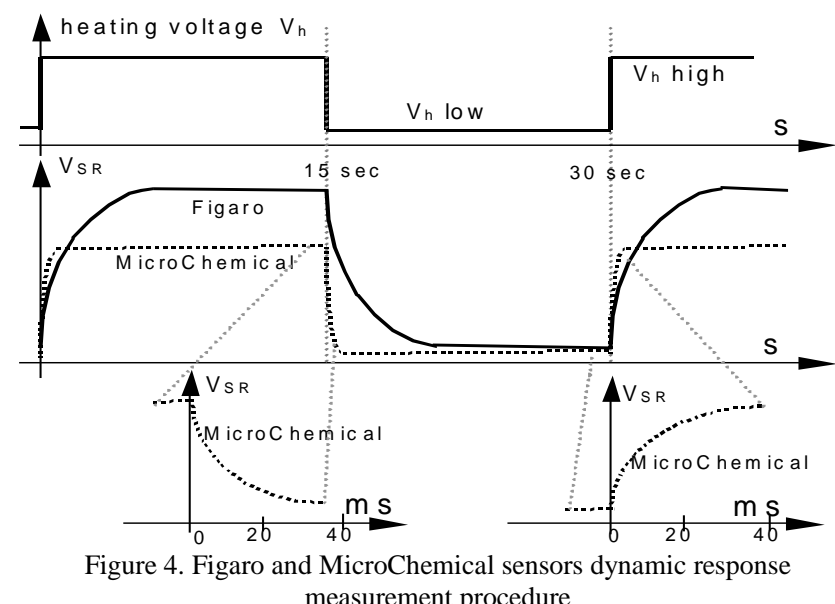

The MicroChemical Systems sensors' steady hot and steady cool phases do not provide much useful information, so we record only their heating and cooling phases, taking 250 samples of each at a $1 \mathrm{kHz}$ sample rate. The Figaro sensors are intended by the manufacturer to be used in a steady hot mode, but we our goal here to study their dynamic responses. To simplify data collection, we sample them also at $1 \mathrm{kHz}$, then average each block of 250 samples, giving an equivalent sampling rate of $4 \mathrm{~Hz}$, which is commensurate with the fastest signal changes or fluctuations they are observed to produce.

\section{DATA AND DATA ANALYSIS}

We report our initial qualitative observations and subsequent quantitative measurements of sensor static response for a variety of samples and sample mixtures. Later dynamic measurements have not yet been fully analyzed.

Preliminary experiments were essentially go/no-go based on whether or not a high but not necessarily calibrated sample concentration produced a notable response. Quantitative measurements of static response vs. concentration were made for those sensor and sample types for which potentially usable response was identified in the qualitative tests ${ }^{1}$.

\footnotetext{
${ }^{1}$ Note, however, that the absence of a response (or the absence of a large response) by a particular sensor to a particular odorant does not necessarily mean that the sensor has no place in a system to detect that odorant. On the contrary, a sensor that is blind to an odorant or environmental component that another sensor sees, can - in a judicious combination of the two - resolve the interferences from odorants to which both sensors are sensitive.
} 


\section{A. Figaro Sensor Static Response}

We examined qualitatively the static response of Figaro sensors TGS825, TGS826, TGS2600 and TGS2620 to the gases and vapors believed to be potential practical signatures for fecal incontinence. Trials included 3\% hydrogen peroxide in water, $1 \%$ ethanol in water, $0.7 \%$ isopropanol in water, $1 \%$ Clorox bleach $(5.25 \%$ sodium hypo chlorite) in water, $0.75 \%$ formalin in water, 3\% household ammonia (3\%) in water, mixture of vapors of dimethyl disulfide, indole, hydrogen sulfide and ammonia, (organoleptically blended to smell like feces), the feces-like mixture with bleach added, the feceslike mixture with isopropanol added, actual urine samples, and actual feces samples.

Interesting cases were followed by static quantitative measurements in which we paid careful attention to controlling sample concentration and humidity. Responses were measured to: indole vapor $(0.49 \mathrm{ppm})$, skatole vapor (0.35 ppm), hydrogen sulfide gas (100 ppm, $10 \mathrm{ppm}$, and 5 $\mathrm{ppm})$, and dimethyl disulfide vapor (0.07 ppm, $0.14 \mathrm{ppm}$, and $0.7 \mathrm{ppm})$.

Comprehensive tabulation of experiment conditions and results for all these experiments is beyond the scope of the present paper. A typical case, four sensors' responses to three concentrations (and one blank) of dimethyl disulfide vapor are shown in Table 2.

Table 2. Figaro sensors' relative change of resistance change $\Delta R_{s} / R_{s}$ vs. dimethyl disulfide vapor concentration

\begin{tabular}{|c|c|c|c|c|}
\hline & TGS825 & TGS826 & TGS2600 & TGS2620 \\
\hline Clean air $\mathrm{R}_{\mathrm{s}}(\mathrm{k} \Omega)$ & $81.6 \sim 92.5$ & $106.6 \sim 108.6$ & $32.8 \sim 39.6$ & $37.3 \sim 45.7$ \\
\hline $0.07 \mathrm{ppm}\left(\Delta R_{s} / R_{s}\right)$ & $14.1 \sim 15.1 \%$ & $18.1 \sim 19.0 \%$ & $\sim 14.9 \%$ & $16.4 \sim 16.6 \%$ \\
\hline $0.14 \mathrm{ppm}\left(\Delta R_{s} / R_{s}\right)$ & $12.2 \sim 15.5 \%$ & $20.9 \sim 25.4 \%$ & $14.6 \sim 17.4 \%$ & $16.4 \sim 19.9 \%$ \\
\hline $0.7 \mathrm{ppm}\left(\Delta R_{s} / R_{s}\right)$ & $25.5 \sim 27.6 \%$ & $58.9 \sim 61.0 \%$ & $29.0 \sim 31.9 \%$ & $32.1 \sim 34.8 \%$ \\
\hline
\end{tabular}

This table illustrates the conclusion that emerges from the full set of qualitative and quantitative experiments: all the Figaro sensors exhibit static responses to all the compounds of interest. The responses are monotonic but nonlinear with concentration. They are quantitatively approximately consistent with the well known exponential model for TGS devices[5]:

$$
\mathrm{R}=\mathrm{R}_{0}\left(\left[\mathrm{O}_{2}\right] /\left(1+\mathrm{K}_{\mathrm{X}}[\mathrm{X}]\right)\right)^{\beta}
$$

where $\mathrm{R}$ is the sensor's resistance, $\mathrm{R}_{0}$ is the sensor's resistance under standard conditions, $\left[\mathrm{O}_{2}\right]$ is the molar concentration or partial pressure of oxygen, $[\mathrm{X}]$ is the molar concentration or partial pressure of chemical species $\mathrm{X}, \mathrm{K}_{\mathrm{X}}$ is a rate constant characteristic of $X$, and $\beta$ is an exponent, typically between 0.25 and 0.55 , that is idiosyncratic to the particular sensor.

At least across the range of odorants that are of obvious potential interest in the context of a fecal incontinence sensor array, there is so little differential selectivity from Figaro TGS model-to-model that a sensor array signature recognition approach does not appear feasible when the sensors are used in the steady heated mode recommended by the manufacturer.

\section{B. Drift and Low Frequency Noise}

Apparently random excursions of sensor resistance sometimes approach $50 \%$, outweighing sensor response to most odorants at anticipated concentrations. Figure 4 shows the resistance drift of Figaro TGS825, TGS826, TGS2600 and TGS2620 sensors over a 24-hour period when they were held in the above described sensor chamber with a 94.4 liter per minute purified and dehumidified compressed air ventilation.

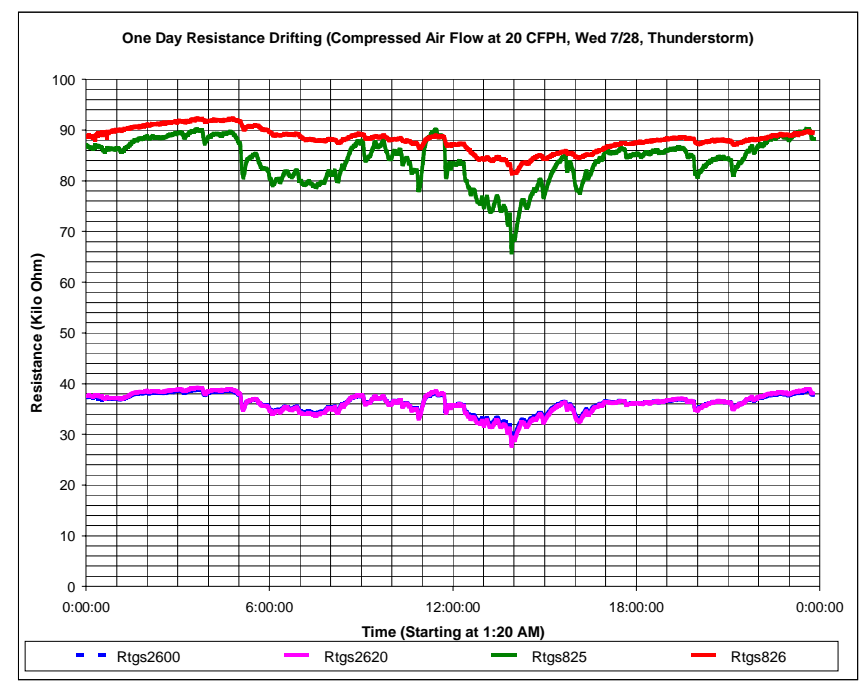

Figure 5. Figaro sensor resistance drift over 24 hour.

In this experiment we diligently tried to keep every controllable factor (heater voltage, airflow, etc.) stable, and we believe we succeeded. The obviously large drift and low frequency noise seen in Figure 5 thus seems attributable only to random processes within the sensor, not to any uncontrolled external influences save possibly one discussed in following section.

\section{Humidity}

The one external influence that we suspect may be insufficiently well controlled is humidity. The sensitive response of the TGS sensor to humidity is illustrated by Figure 6, which shows the sensors' responses to the addition and removal of a $5 \%$ water vapor saturated airflow to the $95 \%$ nominally dry steady airflow. At room temperature, $100 \%$ humidity corresponds to about $2.5 \%$ molar concentration of water, so this response corresponds to a water vapor molar concentration of about $0.125 \%$. 


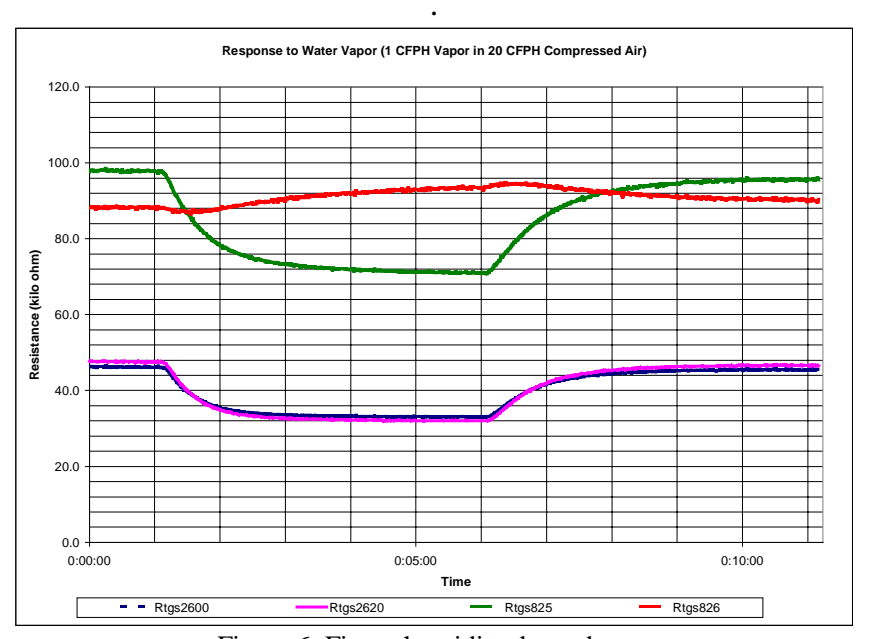

Figure 6. Figaro humidity dependence.

This sensitivity to humidity demonstrates the crucial requirement for humidity compensation in any practical fecal incontinence sensor that utilizes these or similar component sensors.

\section{CONCLUSIONS AND FUTURE WORK}

The sensors' low-frequency noise and drift rule out a fecal incontinence sensor that uses an array of general-purpose metal oxide sensors operated statically: the small flow rates and large volumes of air anticipated in the nursing home scenario would present odor and humidity concentration rates of change that as a practical matter could not be distinguished from sensor drift.

Dynamic response measurements (details not reported herein) seem to be effective at overcoming drift. However cross sensitivity, especially to humidity, remains a computational challenge in the dynamic response case as well. Pattern recognition algorithms to exploit the dynamic response in the presence of cross sensitivity are still being developed. The traditional discrete Figaro sensors and new integrated circuit MicroChemical Systems sensors could both be used. The 150x faster heating and cooling time of the MicroChemical Systems sensors make them seem more promising, but their approximately $5 \mathrm{x}$ greater cost may prove prohibitive in the low cost application scenario envisioned.

We conclude:

- Both Figaro sensors and MicroChemical Systems sensors exhibit very large variation from sensor-tosensor of the same model.

- The sensors' actual selectivity from model-to-model is less dramatic than manufacturer's designations would suggest. The differences from sensor-to-sensor may actually exceed the mean differences from model-tomodel.
- The sensors all have low frequency noise and long-term drift problems. Measuring their dynamic response to temperature programming appears to be a promising alternative. Details of the latter experiments will be reported later.

The complexity of the observed sensor responses and the complication of their extreme sensitivity to humidity means that building a low cost incontinence sensor may not be as easy as we initially anticipated. Our present effort is directed toward a differential design that will employ matched pairs of sensors: one equipped with an activated charcoal filter to trap organic vapors, the other without the filter. Since the filter does not trap humidity, the differential response of these sensors should be due entirely to the organics of interest. Experiments are in progress, and we expect that early results will be available by the conference time.

\section{REFERENCES}

[1] Dr. Roger Luckenbach, Fecal Matters, Here's the poop on poop, http://www. plumbingproducts.com/pooppage.html

[2] Aldrich Chemical Company, Inc., Product Catalog 1996, 1001 West Saint Paul Avenue, Milwaukee, WI 53233, 1-800-558-9160, 1-800771-6737

[3] Figaro USA, INC, General Technical Information, http://www.figarosensor.com, (3703 West Lake Ave. Suite 203, Glenview, Illinois 60025, phone: 1-847-832-1701)

[4] J. G. Moore, L. D. Jessop, and D. N. Osborne, Gas-Chromatographic and Mass-Spectrometric Analysis of the Odor of Human Feces, Gastroenterology Dec., 1987, Vol. 93, No. 6, page 1321-1329.

[5] Mel Siegel, Olfaction Metal Oxide Semiconductor Gas Sensors and Neural Networks, in book Traditional and Non-Traditional Sensors for Robotics, NATO Advanced Research Workshops, volume F63, pages 143-157, Springer-Verlag, Berlin, 1990.

[6] Arnold J. Gordon, Richard A. Ford, The Chemist's Companion: a Handbook of Practical Data, Techniques, and References, John Wiley \& Sons, Inc., 1972.

[7] MicroChemical Systems SA, Rue Porcena 15, CH-2035 Corcelles, Switzerland.See http://www.microchemical.com.

[8] Stephen R. Heller, editor, Beilstein Online Database: Implemention, content, and retrieval, Washington, DC: American Society, 1990 